\title{
EntangleVR: A Visual Programming Interface for Virtual Reality Interactive Scene Generation
}

\author{
Mengyu Chen \\ mengyuchen@ucsb.edu \\ University of California Santa Barbara \\ Santa Barbara, CA, USA
}

\author{
Marko Peljhan \\ peljhan@mat.ucsb.edu \\ University of California Santa Barbara \\ Santa Barbara, CA, USA
}

\author{
Misha Sra \\ sra@cs.ucsb.edu \\ University of California Santa Barbara \\ Santa Barbara, CA, USA
}
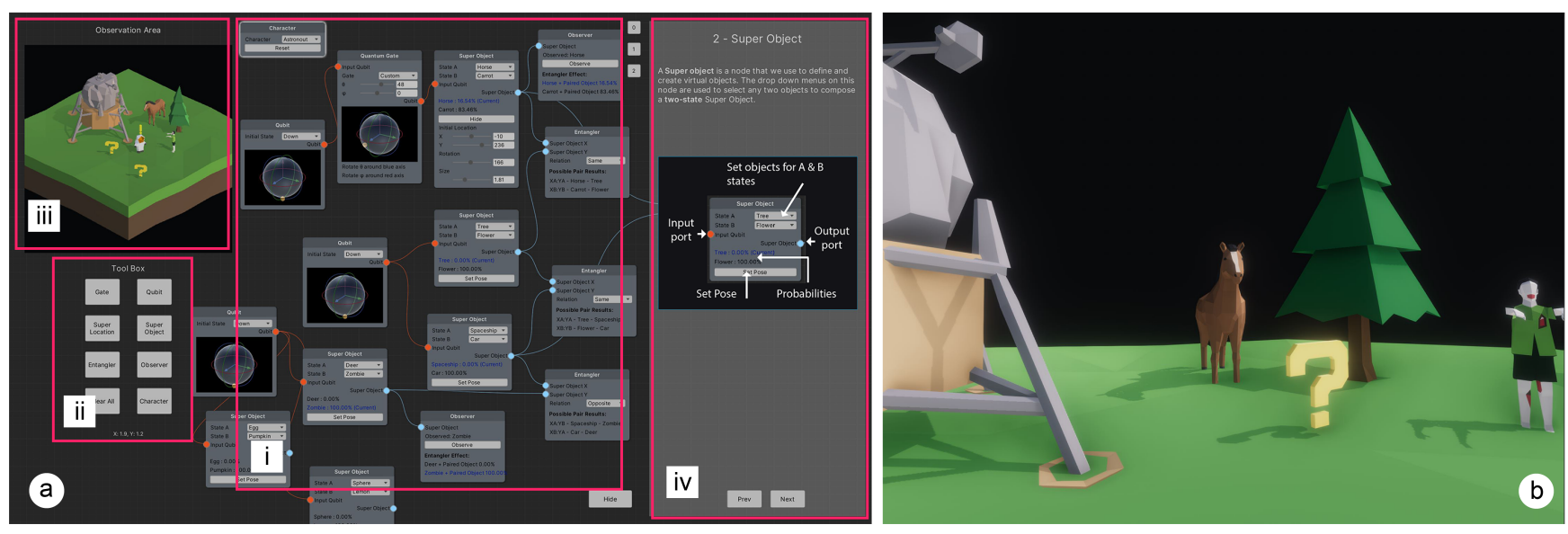

Figure 1: EntangleVR system overview. Left: The visual programming interface where (i) is the main canvas to draw nodebased graphs (ii) is the Toolbox with buttons to create nodes, (iii) is the real-time interactive preview which renders the visual results of the graph, and (iv) is the tutorial panel. Right: (b) shows the first person view of a VR scene created with EntangleVR.

\begin{abstract}
Entanglement is a unique phenomenon in quantum physics that describes a correlated relationship in the measurement of a group of spatially separated particles. In the fields of science fiction, game design, art and philosophy, it has inspired the creation of numerous innovative works. We present EntangleVR, a novel method to create entanglement-inspired virtual scenes with the goal to simplify representing this phenomenon in the design of interactive VR games and experiences. By providing a reactive visual programming interface, users can integrate entanglement into their design without requiring prior knowledge of quantum computing or quantum physics. Our system enables fast creation of complex scenes composed of virtual objects with manipulable correlated behaviors.
\end{abstract}

\section{CCS CONCEPTS}

- Human-centered computing $\rightarrow$ Virtual reality; User interface programming.

Permission to make digital or hard copies of all or part of this work for personal or classroom use is granted without fee provided that copies are not made or distributed for profit or commercial advantage and that copies bear this notice and the full citation on the first page. Copyrights for components of this work owned by others than the author(s) must be honored. Abstracting with credit is permitted. To copy otherwise, or republish, to post on servers or to redistribute to lists, requires prior specific permission and/or a fee. Request permissions from permissions@acm.org.

VRST '21, December 8-10, 2021, Osaka, Japan

(C) 2021 Copyright held by the owner/author(s). Publication rights licensed to ACM. ACM ISBN 978-1-4503-9092-7/21/12 ..\$15.00

https://doi.org/10.1145/3489849.3489872

\section{KEYWORDS}

virtual reality, quantum computing, entanglement, visual programming, art, creativity, 3D scene creation

\section{ACM Reference Format:}

Mengyu Chen, Marko Peljhan, and Misha Sra. 2021. EntangleVR: A Visual Programming Interface for Virtual Reality Interactive Scene Generation. In 27th ACM Symposium on Virtual Reality Software and Technology (VRST '21), December 8-10, 2021, Osaka, Japan. ACM, New York, NY, USA, 6 pages. https://doi.org/10.1145/3489849.3489872

\section{INTRODUCTION}

Quantum computing offers many interesting concepts that can be applied to creative computing like interactive scene creation for virtual reality (VR) or game design and other forms of experiential media. Entanglement, a phenomenon that is at the heart of disparity between classical and quantum physics, describes a correlated relationship between the measurement of multiple spatially separated particles [2,27]. As a unique feature of quantum computing, it transforms individual object states into non-separable shared states, allowing a single interaction on one object's state to affect all the entangled object states as a whole [22].

In the domain of virtual world creation, we believe that the concept of entanglement can empower artists and creators to build narrative structures that incorporate non-separable interactions, events, choices and relationships between virtual objects and create a dynamic scene of shifting patterns. A narrative woven with entangled relationships can potentially simulate real life scenarios more 
realistically where seemingly unrelated events and choices can create substantial but unforeseen impact on others and the world around us. Digital storytelling can be more interaction-driven and more dynamically influenced by the user's conscious or even unconscious choices when user interactions are associated with the state of scene elements in an entangled fashion.

In this work, we present EntangleVR, an interactive system that enables users to create virtual environments using the entanglement concept without requiring prior knowledge of quantum computing or quantum physics. By letting users of our system create "entangled" game objects that have correlated behaviors, EntangleVR makes it easy to simulate complex relationships between objects and entities for building games, VR experiences and other forms of interactive experiences. EntangleVR provides a novel method to create entanglement-based virtual objects for interactive VR scene design using a reactive visual programming interface. The virtual scenes created by our system exhibit non-deterministic quantumlike behavior patterns and the elements in the scenes can be entangled together to formulate shared states controllable by probability distributions. User interactions can participate in this entangled environment and produce an "observer effect" [14] to make the quantum states collapse based on their probabilities and entangled correlations. A collapse occurs when an object initially in multiple possible states reduces to a single state due to interaction with the external world. EntangleVR not only provides a reactive visual programming interface for easy and fast creation, but also includes an instant preview of built virtual environments in VR, where users can switch between graph-based scene composition and immersive viewing without compiling. Our main contributions are:

- A reactive visual programming interface for interactive VR scene generation.

- A set of quantum computing inspired operations, interactions and visual representations for creating probabilistic virtual object behaviors.

\section{RELATED WORK}

EntangleVR is inspired by prior work in VR scene authoring, visual programming for creativity, and quantum computing.

\subsection{VR Scene Authoring}

VR scene authoring tools allow users to create a virtual environment by adding and manipulating virtual objects as well as their behaviors. Prior works have looked at various forms of VR scene authoring and prototyping. We classify two main types: low level and high level approaches. Low level approaches provide programming libraries or frameworks to help build VR applications such as Alice [23], CalVR [28], FreeVR [29] or VRPN [34]. High level approaches are rapid prototyping tools mainly designed for spatial scene creation, 3D user interface, and object interactions that are independent from hardware architecture, operating system or VR device configuration. Saquib et al. [31] proposed a visualized dataflow representation for defining behaviors in virtual environment. VR Juggler [3] and RUIS [33] are frameworks that let user easily build VR applications without necessary technical knowledge regarding the underlying hardware devices. Billinghurst [4] presented 3D Palette, a new interface for rapid 3D virtual scene creation using tablet input, $6 \mathrm{DoF}$ direct input and multi-modal input. More recently, immersive authoring tools are becoming more available by the concept of What-You-eXperience-Is-What-You-Get (WYXIWYG) [16]. ISAAC [19] is one of the earliest authoring tools to support interactive construction of virtual worlds directly in an immersive setup. 360proto [21] allows user to rapidly create $\mathrm{AR} / \mathrm{VR}$ prototypes from paper and have direct access to the device view during editing process. FlowMatic [39] proposed a reactive visual programming tool to program and create interactive scenes in VR. Taking inspirations from both high level approaches and immersive authoring, EntangleVR proposes a hybrid approach of scene authoring by providing a $2 \mathrm{D}$ visual programming interface for rapid prototyping while allowing an instant and interactive preview of created VR scenes without compiling or building.

\subsection{Visual Programming for Creativity}

Visual programming systems provide an approach and methods that use graphical elements to compose a program [20]. Data or algorithms are often visualized to show how the composed program operates. The visual representation of programming constructs such as variable setting, control flow and conditionals are well integrated for learning and education environments to promote creativity $[6,15,17]$. The advantages of low barrier and simplicity have also led to many content creation software and platforms to adopt visual programming as the main composition method for artists and designers. For example, creators in various professional fields such as game development [7], film production [30], architecture [26], music [10], and large-scale art installation [35, 36], use visual programming tools in their daily work. In research, previous works have explored the potential use of visual programming tools in different creative domains such as media arts pedagogy [8], digital art exhibitions [32], 3D interactive graphics [9], and digital storytelling [12]. EntangleVR similarly aims to support creativity with its easy to use visual programming interface. It is designed to target beginner users for VR game development and media arts.

\subsection{Quantum Computing for Creativity and Learning}

The quantum phenomenon and concepts have also been widely gamified and the creative community has gained rich inspiration from quantum science. Quantum Moves [25] is an online platform gamifying optimization problems in quantum physics where players move simulated quantum atoms to create optimal control solutions for real atoms. Hamido et. al. [13] proposed a quantum computing based music synthesizer that maps quantum circuits into music scores. Quantum Game with Photons 2 [24] is an online drag and drop puzzle game that visualizes the photons as light waves with forms drawn from quantum equations. Anupam et al. [1] presented a digital game design process and case study for classrooms which involves and supplements introductory quantum mechanics curricula. Other educational tools to teach the basics of quantum computing have emerged in formats such as board games [37] and 3D puzzles [11]. Recent work by Zable et. al. [38] investigates using VR as an educational tool for quantum computing, allowing users to interact with a 3D Bloch sphere visualization of a qubit. EntangleVR not only looks at how quantum computing 

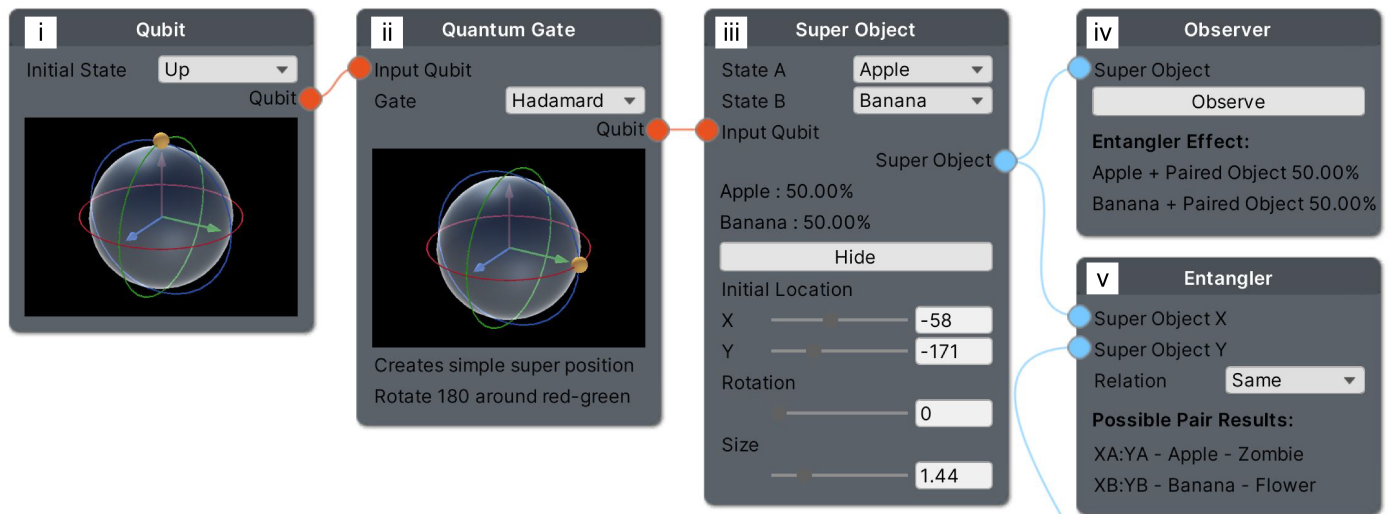

Figure 2: EntangleVR's quantum-inspired node design. Left: (i) is a Qubit node in an initial state of $|0\rangle$ (Up). Left-center: (ii) is a Gate node set as a Hadamard gate. Right-center: (iii) is a Super Object node with a $50 \%$ probability of turning into either state A (apple) or state B (banana). Right: (iv) is an Observer node reflecting the expected measurement outcome of input super object. (v) is an Entangler node that entangles two super object together by a user-set relation.

and its related concepts can benefit content creators in VR, but also aims to help its users learn new computational thinking concepts from the quantum domain through encapsulation of concepts in the visual interface.

\section{SYSTEM DESIGN}

The EntangleVR visual programming environment is built as a plugin for the Unity game engine with a node-based graph interface and a custom-built quantum simulator written with Math.NET library[18] to enable basic quantum operations and support quantumlike indeterminacy. The system allows the user to create different nodes that allow composing interactive virtual environments. Immediate visual feedback is provided through the composed scene as and when the node parameters are modified.

\subsection{User Interface}

EntangleVR aims to provide an easy and straightforward interface for VR content creators. The interface has a single window containing all the key components where users can select and create nodes, compose graphs, and interact with the visual output, all at the same time. Figure 1 shows the EntangleVR interface, which has four main panels. The lower left panel is the Toolbox with buttons to create nodes. The middle panel is the graph canvas where created nodes appear and can be connected to formulate a graph. The upper left panel is an interactive preview which renders the visual results of the graph in real-time as the nodes are being edited. The rightmost panel contains a tutorial and step-by-step walkthrough of the interface as well as the integrated quantum concepts. This can be turned on/off by the user at will.

\subsection{Quantum-inspired Node Design}

EntangleVR enables users to create 3D virtual environments by connecting nodes together to formulate a relational graph of virtual objects. The nodes in EntangleVR can represent object instances, logical statements, and control events. All the nodes have input ports on the left and output ports on the right. The ports are typeconstrained and their colors inform the user if they can be linked together. There are seven types of nodes in EntangleVR: Super Object, Qubit, Gate, Observer, Entangler, Super Location, and Avatar. They are all inspired by either quantum computing or quantum phenomena and contribute to the probabilistic quantum-like behaviors of the virtual objects. Figure 2 shows a selected set of nodes in EntangleVR system.

3.2.1 Qubit. A qubit node provides the basic unit of computation same as the one in quantum computing. To aid comprehension, we include an interactive Bloch Sphere representation [5] in the node block to immediately depict the states of a qubit with a clear geometric reference to its basis vectors such as "Zero", $|1\rangle$ as "One", $|+\rangle$ as "Super Plus", $|-\rangle$ as "Super Minus" [37].

3.2.2 Super Object. This node is a higher-level mapping of a qubit's computational basis $(|0\rangle$ and $|1\rangle)$ into virtual objects. A two-state object is designed to simulate the uncertain super-position nature of a qubit, and the created super object by default has a $50 \%$ chance to be measured or observed at state $A$ (e.g., a banana) and $50 \%$ chance to be in state $B$ (e.g., an apple). A qubit can be fed into a super object as input to replace and change the probabilities of the two states using the qubit's probability amplitudes. Once this node is created, a super object icon appears as a question mark in the preview panel since it can be in either of the two states. The user can move its position, orientation and scale by changing its parameters.

3.2.3 Observer. An observer node provides an interaction method to measure and collapse the linked super object i.e., set it's state to A or B, using a default computational basis $|0\rangle$ and $|1\rangle$. The user can choose to observe the super object and turn it into a classical state based on the amplitudes of states A and B. Observation can be triggered by three interaction methods: 1) VR mode - touching the super object with a virtual hand and clicking the trigger button on the VR controller, 2) 2D mode - in the 2D observation window, controlling the virtual character's avatar to approach the super object and double-clicking the super object, and 3) Preview mode - 

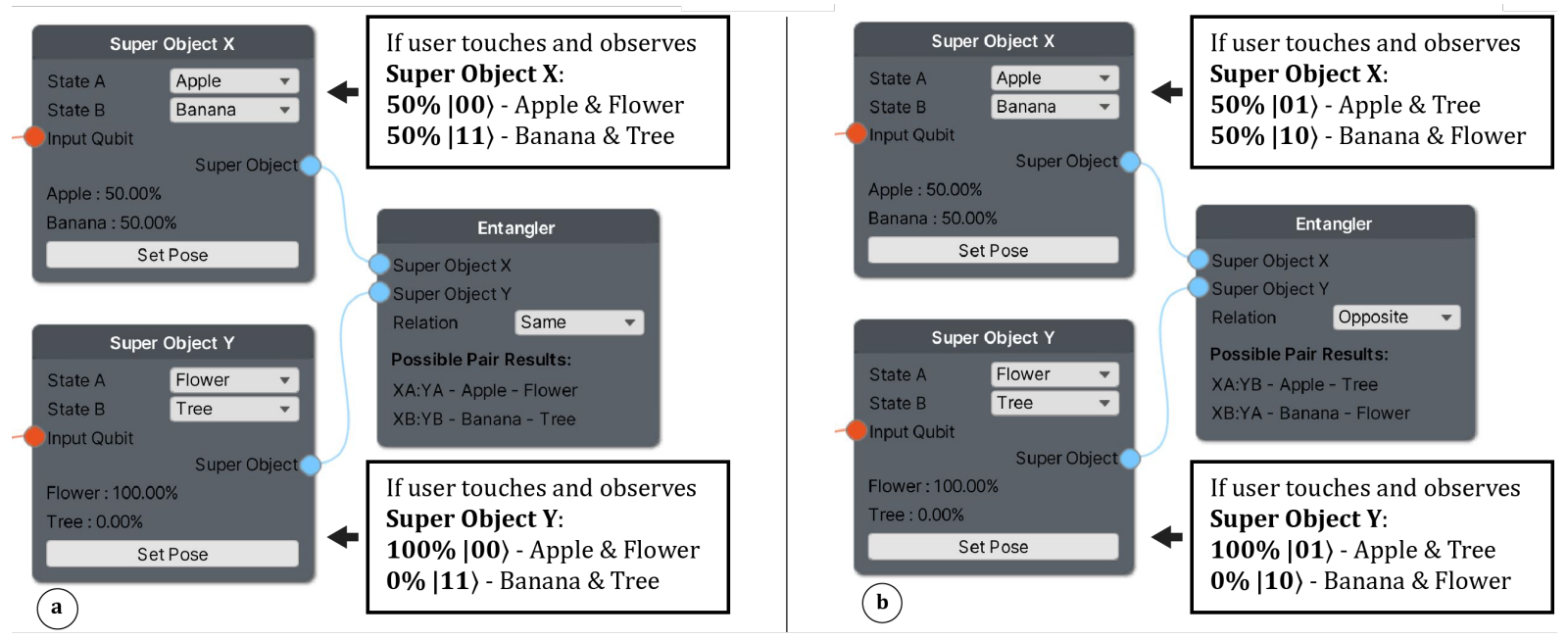

Figure 3: An example of how the entanglement effect between two super objects of different probability amplitudes can be played by the user interaction. Left: (a) shows the "Same" effect leading to a $|00\rangle$ or $|11\rangle$ state. Right: (b) shows the "Opposite" effect leading to a $|01\rangle$ or $|10\rangle$ state.

clicking the "Observe" button on the node block. Upon observation, the super object, instead of showing as a question mark, will change its rendered icon to one of the two states (e.g., apple or banana).

3.2.4 Gate. A gate node provides a selected set of common singlequbit quantum gates such as Hadamard gate, Pauli $\mathrm{X}$ gate, and Phase T gate. These logic gates allows the user to manipulate the qubit state and interactively change the amplitudes of the target qubit via the visual interface.

3.2.5 Entangler. The entangler is built to simulate the unique quantum phenomenon of entanglement that is not available (but can be simulated) in the classical computing domain. An entangler node links two super objects (in total four possible states) and creates a non-separable and always-correlated relationship between them when one of the super objects is observed i.e., it's state is set. User can select between the two expressions ("Same" mode and "Opposite" mode) on an entangler. That is to say, user can give this non-separable state different amplitudes based on their interaction choice with one of the super objects. In VR mode, for example, the user can approach and interact with any super object. As soon as the user chooses a super object as their target of observation, an "observer effect" gets triggered on both the entangled super objects. The probability amplitudes of this user-selected super object's qubit (the main qubit) will be used as the amplitudes for state $|00\rangle$ and state $|11\rangle$ (or during "Opposite" mode for state $|01\rangle$ and $|10\rangle)$. Eventually the two qubits "collapse" simultaneously into correlated classical states depending on the probability amplitudes of the main qubit. Figure 3 shows an example of how user interaction can play with the entanglement effect. Please also see the Appendix for example usage of entangler for scene composition.

\subsection{Probabilistic Control over Virtual Objects}

In contrast to existing VR scene authoring systems, EntangleVR uses probability distributions to control virtual objects. Users create super objects to compose the virtual environment and these super objects, including their properties, behave probabilistically based on the state of the driving qubit. By providing a handle over these driving qubits via gates and the entangler, a user can create a state of controllable randomness defined by the probability amplitudes that they can set on the super objects. This can help simulate outcomes more akin of what might happen in real world scenarios where outcomes with $100 \%$ certainty are often unknown. At the same time, switching to a measurement in different basis other than the default computational basis can dynamically shift all the probability distributions in the entire virtual environment, allowing more creative play and control.

\subsection{Interaction with Chained Object States}

A unique feature of EntangleVR is that it bridges user interaction with properties of quantum-like super objects. For example, observation, as defined in quantum physics, is mapped to user interaction and can be used as a trigger to determine one or multiple virtual object states. This unlocks the potential for mapping multiple types of interactions (e.g., proximity to object could trigger one outcome while picking up the same object could trigger a chain reaction) where the outcome can be easily manipulated through changing probabilities of the super objects and their relationships with entanglement. By using this feature, chained effects can be easily achieved as the result of an observation varies based on the entry point (the first super object to be observed). In EntangleVR, a super object can be simultaneously entangled with many other super objects. An observation on any one of them can trigger chained collapse on all the rest objects. The user can therefore create a mini "butterfly effect" situation with a high number of pattern combinations and possibilities by introducing many participating entangled elements. 


\section{CONCLUSION}

In this work we presented EntangleVR, a novel method to create 3D virtual scenes with entangled objects via a reactive visual programming interface. The concept of entanglement, inspired by quantum physics, enables a new way of defining object relationships and allows the user to easily create a non-deterministic virtual scene. In the generated scenes, user interaction controls the collapse of objects leading to a chain reaction of varying object behaviors in the virtual environment. Our main focus in this work has been to explore the possibility of making complex VR scene creation easier by using the idea of quantum entanglement. We demonstrated EntangleVR's capability to allow beginner users to build relatively complex relational virtual scenes through a visual programming interface. In the future, we will build upon the encouraging results from our interface usability study $(n=16)$ with a design study involving artists and storytellers. We will also expand our system by adding entanglement at different scene creation scales (e.g., procedural scene generation, multi-scene entanglement).

\section{REFERENCES}

[1] Aditya Anupam, Ridhima Gupta, A. Naeemi, and Nassim Jafarinaimi. 2018. Particle in a Box: An Experiential Environment for Learning Introductory Quantum Mechanics. IEEE Transactions on Education 61 (2018), 29-37.

[2] John S. Bell. 1964. On the Einstein-Podolsky-Rosen paradox. Physics Physique Fizika 1 (1964), 195-200.

[3] A. Bierbaum, C. Just, P. Hartling, K. Meinert, A. Baker, and C. Cruz-Neira. 2001 VR Juggler: a virtual platform for virtual reality application development. In Proceedings IEEE Virtual Reality 2001. 89-96. https://doi.org/10.1109/VR.2001. 913774

[4] Mark Billinghurst, Sisinio Baldis, Lydia Matheson, and Mark Phillips. 1997. 3D Palette: A virtual reality content creation tool. ACM Symposium on Virtual Reality Software and Technology, Proceedings, VRST, 155-156. https://doi.org/10.1145/ 261135.261163

[5] Felix Bloch. 1946. Nuclear Induction. Physical Review 70, 7-8 (Oct. 1946), 460-474 https://doi.org/10.1103/PhysRev.70.460 Provided by the SAO/NASA Astrophysics Data System.

[6] Blockly 2021. Blockly: Games for tomorrow's programmers. Retrieved July 11 2021 from https://blockly.games/

[7] Blueprint VIsual Scripting 2021. Blueprint Visual Scripting: OVerview of using the Blueprint visual scripting system for gameplay. Retrieved July 11, 2021 from https: //docs.unrealengine.com/4.26/en-US/ProgrammingAndScripting/Blueprints/

[8] Scott Andrew Brown, Michael Gratton, and Bronwen Williams. 2019. A Visual Programming Tool for Creative Practice Pedagogy in Embodied Interaction and Media Arts. In Proceedings of the 31st Australian Conference on HumanComputer-Interaction (Fremantle, WA, Australia) (OZCHI'19). Association for Computing Machinery, New York, NY, USA, 364-368. https://doi.org/10.1145/ 3369457.3369495

[9] Alberto Cannavò, Francesco De Pace, Federico Salaroglio, and Fabrizio Lamberti 2020. A visual editing tool supporting the production of 3D interactive graphics assets for public exhibitions. International fournal of Human-Computer Studies 141 (2020), 102450. https://doi.org/10.1016/j.ijhcs.2020.102450

[10] Cycling'74 Max 2021. Max is software for experimentation and invention. Retrieved July 11, 2021 from https://cycling74.com/

[11] Simon J. Devitt. 2016. Programming quantum computers using 3-D puzzles, coffee cups, and doughnuts. arXiv:1609.06628 [quant-ph]

[12] Henrik Engström, Jenny Brusk, and Patrik Erlandsson. 2018. Prototyping Tools for Game Writers. The Computer Games fournal 7 (09 2018). https://doi.org/10. 1007/s40869-018-0062-y

[13] Omar Costa Hamido, Giovanni Amedeo Cirillo, and Edoardo Giusto. 2020. Quantum Synth: A Quantum-Computing-Based Synthesizer. In Proceedings of the 15th International Conference on Audio Mostly (Graz, Austria) (AM '20). Association for Computing Machinery, New York, NY, USA, 265-268. https: //doi.org/10.1145/3411109.3411135

[14] Werner Heisenberg. 1958. Physics and Philosophy: The Revolution in Modern Science. New York: Harper.

[15] Anastasia Kovalkov, Avi Segal, and Kobi Gal. 2020. Inferring Creativity in Visual Programming Environments. In Proceedings of the Seventh ACM Conference on Learning @ Scale (Virtual Event, USA) (L@S '20). Association for Computing Machinery, New York, NY, USA, 269-272. https://doi.org/10.1145/3386527.3406725
[16] Gun A. Lee, Gerard J. Kim, and Mark Billinghurst. 2005. Immersive Authoring: What You EXperience Is What You Get (WYXIWYG). Commun. ACM 48, 7 (July 2005), 76-81. https://doi.org/10.1145/1070838.1070840

[17] John Maloney, Mitchel Resnick, Natalie Rusk, Brian Silverman, and Evelyn Eastmond. 2010. The Scratch Programming Language and Environment. ACM Trans. Comput. Educ. 10, 4, Article 16 (Nov. 2010), 15 pages. https://doi.org/10.1145/ 1868358.1868363

[18] Math.NET 2021. Math.NET Numerics. Retrieved July 11, 2021 from https:// numerics.mathdotnet.com/

[19] Mark R. Mine. 1995. ISAAC: A Virtual Environment Tool for the Interactive Construction of Virtual Worlds. Technical Report. USA.

[20] Brad A. Myers. 1990. Taxonomies of visual programming and program visualization. Journal of Visual Languages and Computing 1, 1 (1990), 97-123. https://doi.org/10.1016/S1045-926X(05)80036-9

[21] Michael Nebeling and Katy Madier. 2019. 360proto: Making Interactive Virtual Reality \& Augmented Reality Prototypes from Paper. Proceedings of the 2019 CHI Conference on Human Factors in Computing Systems (2019).

[22] Michael A. Nielsen and Isaac L. Chuang. 2010. Quantum Computation and Quantum Information: 10th Anniversary Edition. Cambridge University Press.

[23] Randy Pausch, Tommy Burnette, AC Capeheart, Matthew Conway, Dennis Cosgrove, Rob DeLine, Jim Durbin, Rich Gossweiler, Shuichi Koga, , and Jeff White. 1995. Alice: rapid prototyping for virtual reality. IEEE Computer Graphics and Applications 15, 3 (1995), 8-11. https://doi.org/10.1109/38.376600

[24] Quantum Game with Photons 2 2021. Quantum Gane with Photons: An in-browser simulation and visualization of quantum mechanics. Retrieved July 11, 2021 from https://quantumgame.io/

[25] Quantum Moves 2021. Quantum Moves: Help scientist by simulating logical operations in a quantum computer. Retrieved July 11, 2021 from https: //citizensciencegames.com/games/quantum-moves/

[26] Rhinoceros 2021. Grasshopper. Retrieved July 11, 2021 from https://www.rhino3d. com/6/new/grasshopper/

[27] Erwin Schrödinger. 1935. Discussion of Probability Relations between Separated Systems. Mathematical Proceedings of the Cambridge Philosophical Society 31, 4 (1935), 555-563.

[28] Jürgen Schulze, Andrew Prudhomme, Philip Weber, and Thomas DeFanti. 2013. CalVR: An Advanced Open Source Virtual Reality Software Framework. Proceedings of SPIE - The International Society for Optical Engineering 8649 (03 2013), 02-. https://doi.org/10.1117/12.2005241

[29] William R. Sherman, Daniel Coming, and Simon Su. 2013. FreeVR: honoring the past, looking to the future. In The Engineering Reality of Virtual Reality 2013 (Society of Photo-Optical Instrumentation Engineers (SPIE) Conference Series, Vol. 8649), Margaret Dolinsky and Ian E. McDowall (Eds.).

[30] SideFX 2021. Houdini Overview. Retrieved July 11, 2021 from https://www.sidefx. com/products/houdini/

[31] Anthony Steed and Mel Slater. 1996. A dataflow representation for defining behaviours within virtual environments. In Proceedings of the IEEE 1996 Virtual Reality Annual International Symposium. 163-167. https://doi.org/10.1109/VRAIS. 1996.490524

[32] Andrew Stratton, Chris Bates, and Andy Dearden. 2017. Quando: Enabling Museum and Art Gallery Practitioners to Develop Interactive Digital Exhibits. In End-User Development, Simone Barbosa, Panos Markopoulos, Fabio Paternò, Simone Stumpf, and Stefano Valtolina (Eds.). Springer International Publishing, 100-107.

[33] Tuukka Takala. 2014. RUIS - A toolkit for developing virtual reality applications with spatial interaction. SUI 2014 - Proceedings of the 2nd ACM Symposium on Spatial User Interaction. https://doi.org/10.1145/2659766.2659774

[34] Russell M. Taylor, Thomas C. Hudson, Adam Seeger, Hans Weber, Jeffrey Juliano, and Aron T. Helser. 2001. VRPN: A Device-Independent, Network-Transparent VR Peripheral System. In Proceedings of the ACM Symposium on Virtual Reality Software and Technology (Baniff, Alberta, Canada) (VRST '01). Association for Computing Machinery, New York, NY, USA, 55-61. https://doi.org/10.1145/ 505008.505019

[35] TOUCHDESIGNER by Derivative 2021. TOUCHDESIGNER. Retrieved July 11, 2021 from https://derivative.ca/

[36] vvvv 2021. $v v v v$ - a multipurpose toolkit. Retrieved July 11, 2021 from https: //vvvv.org/

[37] Justin D. Weisz, Maryam Ashoori, and Zahra Ashktorab. 2018. Entanglion: A Board Game for Teaching the Principles of Quantum Computing (CHI PLAY '18). Association for Computing Machinery, New York, NY, USA, 523-534. https: //doi.org/10.1145/3242671.3242696

[38] Alexander Zable, Lloyd Hollenberg, Eduardo Velloso, and Jorge Goncalves. 2020. Investigating Immersive Virtual Reality as an Educational Tool for Quantum Computing. In 26th ACM Symposium on Virtual Reality Software and Technology (Virtual Event, Canada) (VRST '20). Association for Computing Machinery, New York, NY, USA, Article 6, 11 pages. https://doi.org/10.1145/3385956.3418957

[39] Lei Zhang and Steve Oney. 2020. FlowMatic: An Immersive Authoring Tool for Creating Interactive Scenes in Virtual Reality. Association for Computing Machinery, New York, NY, USA, 342-353. https://doi.org/10.1145/3379337.3415824 


\section{A APPENDIX}

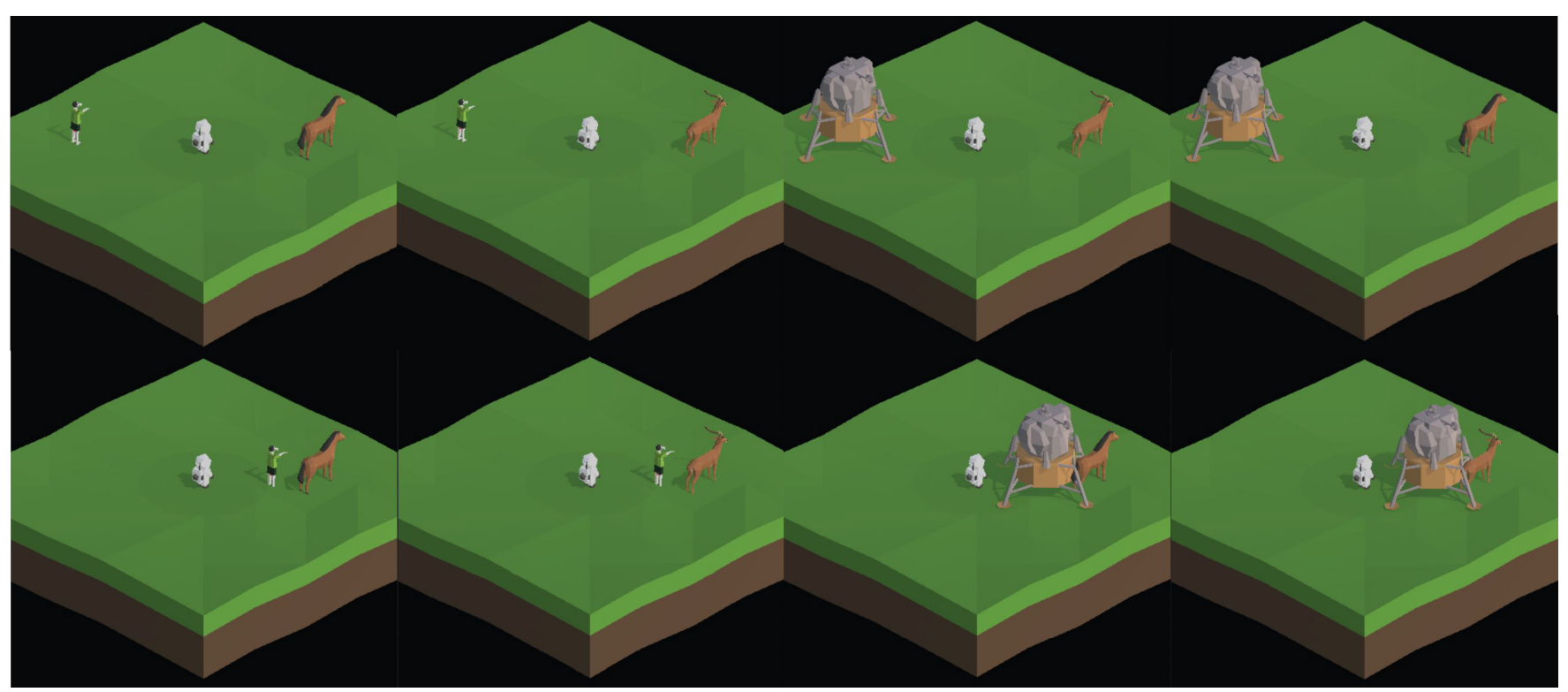

Figure 4: Example scene output from our interface usability study where participants created two super object nodes and one super location node for a simple scene composition. Each created node has probabilities to flip between two custom states. As shown here, there is a combination of eight possible outcomes by using the three nodes (super object $A$ is composed of a horse and a deer, super object $B$ is made of a zombie and a spaceship, and a super location node that causes super object $B$ to appear to the left or the right the astronaut character).

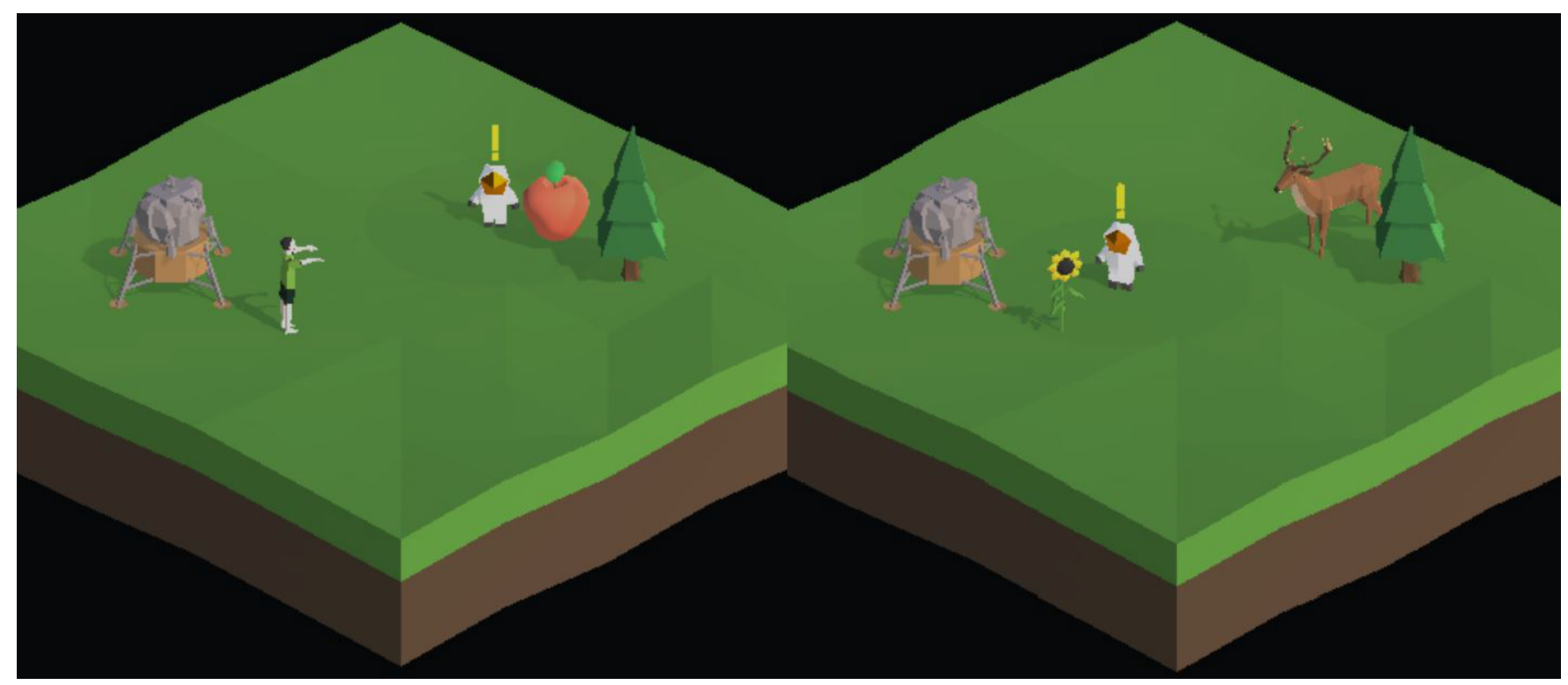

Figure 5: Visual outcomes from our interface usability study where participants were asked to create a virtual environment with entangled objects. The apple and the flower in this scene cannot appear at the same time based on their entangled relationship as created by the participant. The relationships are such that when the virtual human character touches the apple, a zombie appears and destroys the flower, and when the flower is touched, a deer appears and eats the apple. 